PROCEEDINGS OF THE

AMERICAN MATHEMATICAL SOCIETY

Volume 128, Number 2, Pages 463-473

S 0002-9939(99)05075-3

Article electronically published on September 27, 1999

\title{
OBLIQUE PROJECTIONS, BIORTHOGONAL RIESZ BASES AND MULTIWAVELETS IN HILBERT SPACES
}

\author{
WAI-SHING TANG
}

(Communicated by David R. Larson)

\begin{abstract}
In this paper, we obtain equivalent conditions relating oblique projections to biorthogonal Riesz bases and angles between closed linear subspaces of a Hilbert space. We also prove an extension theorem in the biorthogonal setting, which leads to biorthogonal multiwavelets.
\end{abstract}

\section{INTRODUCTION}

In recent developments in wavelet analysis, many significant results have been obtained using the powerful tool of Fourier transform on the Hilbert space $L^{2}\left(\mathbf{R}^{d}\right)$ of square integrable complex-valued functions on $\mathbf{R}^{d}$. However, it is not clear whether analogous results are still valid in domains, other than $L^{2}\left(\mathbf{R}^{d}\right)$, where Fourier transform is no longer available.

In an effort to get a better understanding of the problem, in a series of papers ([5], [9] and [6]) we try to extract the essence of the underlying problem by considering wavelets in a general Hilbert space. This paper is another step in this direction. A recent paper [3] by Dai and Larson is in the same spirit, though with different emphasis.

One of the earliest papers concerning wavelets in Hilbert spaces is that by J. B. Robertson [10], before the birth of modern wavelet theory. While our papers [9] and [6] can be viewed as an extension of the results in [10], the present paper is in turn an extension of [9] and [6] in the following respect. Theorem 2 in [10] leads to orthonormal wavelets associated with orthonormal multiresolutions, whereas an analogous result, [6, Theorem 2.5] (see also [9, Theorem 3.2]), leads to prewavelets. One of our main results in this paper, namely Theorem 3.6, leads to biorthogonal wavelets associated with biorthogonal multiresolutions.

We recall some definitions and set up some notation. Throughout this paper, $H$ denotes a complex Hilbert space. A sequence $\left\{v_{n}\right\}$ in $H$ is a Riesz basis for its closed linear span $V:=\overline{\operatorname{span}}\left\{v_{n}\right\}$ if there exist positive constants $A$ and $B$ such that

$$
A \sum\left|a_{n}\right|^{2} \leq\left\|\sum a_{n} v_{n}\right\|^{2} \leq B \sum\left|a_{n}\right|^{2}, \quad \forall\left\{a_{n}\right\} \in \ell^{2}(\mathbf{Z}) .
$$

Two sequences $\left\{v_{n}\right\}$ and $\left\{\tilde{v}_{n}\right\}$ in $H$ are biorthogonal if

$$
\left\langle v_{n}, \tilde{v}_{m}\right\rangle=\delta_{n, m} \quad \forall n, m .
$$

Received by the editors March 23, 1998.

2000 Mathematics Subject Classification. Primary 46C99, 47B99, 46B15.

Key words and phrases. Riesz basis, biorthogonal system, oblique projection, multiwavelets. 
Chapters 1 and 4 of [12] provide much information on these concepts.

If $V$ and $W$ are closed linear subspaces of $H$ such that $V \cap W=\{0\}$ and $V+W=H$, then we write $H=V \oplus W$ and call this a direct sum. In this case, we can define a map $P: H \longrightarrow H$ by

$$
P(v+w)=v, \quad v \in V, w \in W,
$$

and call $P$ the projection (sometimes oblique projection) of $H$ on $V$ along $W$. For the special case when $W=V^{\perp}$, the orthogonal complement of $V$ in $H$, we shall call $P$ the orthogonal projection of $H$ on $V$, which is related to the orthogonal direct sum $V \oplus^{\perp} V^{\perp}$. Theorem 2.3, a main result in this paper, gives equivalent conditions relating oblique projections to biorthogonal Riesz bases and angles between closed linear subspaces of $H$.

We now give a summary of the contents of this paper. In Section 2, we discuss various relations between oblique projections and biorthogonal Riesz bases. In Section 3, in the biorthogonal setting we obtain an extension theorem (Theorem 3.6), which leads to the existence of biorthogonal multiwavelets. The final section describes briefly the construction of biorthogonal wavelets associated with biorthogonal scaling vectors.

\section{Oblique projections And Biorthogonality}

Let us first state a probably folk result on the vector sum of two closed linear subspaces of a Hilbert space. We leave its proof to the reader.

Theorem 2.1. Let $V$ and $W$ be closed linear subspaces of a complex Hilbert space H. The following conditions are equivalent:

(i) $\sup \{|\langle v, w\rangle|: v \in V, w \in W,\|v\|=\|w\|=1\}<1$.

(ii) There exists a positive constant $C$ such that

$$
\|v+w\|^{2} \geq C\left(\|v\|^{2}+\|w\|^{2}\right), \quad v \in V, w \in W .
$$

(iii) $\quad V+W$ is closed in $H$, and $V \cap W=\{0\}$.

Moreover, if $V \cap W=\{0\}$, and $X$ and $Y$ are Riesz bases for $V$ and $W$ respectively, then (i), (ii) and (iii) are each equivalent to:

(iv) $\quad X \cup Y$ is a Riesz basis for $\overline{V+W}$.

The proof of (iii) $\Longrightarrow(\mathrm{i})$ in the above theorem is implicit in [4, pp. 339-340]. Note that there exist closed linear subspaces $V$ and $W$ of a Hilbert space $H$ for which $V \cap W=\{0\}$, but $V+W$ is not closed in $H$ (see [7, pp. 28-29]).

Corollary 2.2. Let $U_{1}$ and $U_{2}$ be closed linear subspaces of $H$ and $U_{1} \cap U_{2}=\{0\}$. If $U_{1}+U_{2}$ is closed, then $V_{1}+V_{2}$ is closed for any closed linear subspace $V_{i}$ of $U_{i}, i=1,2$.

The expression in condition (i) of Theorem 2.1 is closely related to the concept of the angle $\theta(V, W)\left(0 \leq \theta(V, W) \leq \frac{\pi}{2}\right)$ between two closed linear subspaces $V$ and $W$ of a Hilbert space $H$, which is defined by

$$
\cos (\theta(V, W)):=\inf _{\substack{v \in V \\\|v\|=1}}\left\|P_{W} v\right\|
$$

where $P_{W}$ is the orthogonal projection of $H$ on $W$. (See [1], [4, pp. 339-340] and [11] for related properties and engineering interpretations of $\theta(V, W)$.) It is easy to 
see that

$$
\cos ^{2}(\theta(V, W))=1-\sup _{\substack{v \in V, w \in W \perp \\\|v\|=\|w\|=1}}|\langle v, w\rangle|^{2} .
$$

Note that in general $\theta(V, W)$ and $\theta(W, V)$ are not necessarily equal, but by (2.2) we always have $\theta(V, W)=\theta\left(W^{\perp}, V^{\perp}\right)$. If $V \oplus W^{\perp}=H$, then $\theta(V, W)=\theta(W, V)$.

Theorem 2.3. Let $V$ and $\tilde{V}$ be closed linear subspaces of $H$. The following conditions are equivalent:

(i) $\quad V \oplus \tilde{V}^{\perp}=H$.

(ii) $\quad \tilde{V} \oplus V^{\perp}=H$.

(iii) There exist Riesz bases $\left\{v_{n}\right\}$ and $\left\{\tilde{v}_{n}\right\}$ for $V$ and $\tilde{V}$ respectively such that $\left\{v_{n}\right\}$ is biorthogonal to $\left\{\tilde{v}_{n}\right\}$.

(iv) $\cos (\theta(V, \tilde{V}))>0$ and $\cos (\theta(\tilde{V}, V))>0$.

Proof. (iii) $\Longrightarrow\left(\right.$ ii): Suppose that $\left\{v_{n}: n \in \mathbf{Z}\right\}$ and $\left\{\tilde{v}_{n}: n \in \mathbf{Z}\right\}$ are Riesz bases for $V$ and $\tilde{V}$ respectively such that

$$
\left\langle v_{n}, \tilde{v}_{k}\right\rangle=\delta_{n, k}, \quad n, k \in \mathbf{Z} .
$$

Then

$$
V=\left\{f \in H: f=\sum a_{n} v_{n}, \sum\left|a_{n}\right|^{2}<\infty\right\}
$$

and

$$
\tilde{V}=\left\{g \in H: g=\sum b_{n} \tilde{v}_{n}, \sum\left|b_{n}\right|^{2}<\infty\right\} .
$$

Let $g \in \widetilde{V} \cap V^{\perp}$. By (2.5) and (2.3),

$$
g=\sum\left\langle g, v_{n}\right\rangle \tilde{v}_{n}=0 .
$$

Hence $\widetilde{V} \cap V^{\perp}=\{0\}$.

Let $f \in H$. By the Riesz basis property of $\left\{v_{n}\right\}$ and $\left\{\tilde{v}_{n}\right\}$,

$$
P f:=\sum\left\langle f, v_{n}\right\rangle \tilde{v}_{n}
$$

is a well-defined vector in $\tilde{V}$. By (2.3) and (2.6),

$$
\left\langle f-P f, v_{k}\right\rangle=0, \quad k \in \mathbf{Z} \text {. }
$$

Hence $f-P f \in V^{\perp}$, and so

$$
f=P f+(f-P f) \in \widetilde{V}+V^{\perp} .
$$

Therefore $\widetilde{V}+V^{\perp}=H$.

(ii) $\Longrightarrow$ (iii): Suppose that $\widetilde{V} \oplus V^{\perp}=H$. Let $\left\{v_{n}\right\}$ and $\left\{u_{n}\right\}$ be dual Riesz bases for $V$, i.e., $\left\{v_{n}\right\}$ and $\left\{u_{n}\right\}$ are both Riesz bases for $V$ and

$$
\left\langle v_{n}, u_{k}\right\rangle=\delta_{n, k}, \quad \forall n, k .
$$

Recall that ([12], p. 185 and p. 188) if $F: V \longrightarrow V$ is the frame operator defined by

$$
F(v)=\sum\left\langle v, v_{n}\right\rangle v_{n}, \quad v \in V
$$

then

$$
u_{n}=F^{-1}\left(v_{n}\right), \quad \forall n
$$


Consider the map $P_{V}: H \longrightarrow H$ defined by

$$
P_{V} f:=\sum\left\langle f, v_{n}\right\rangle u_{n}, \quad f \in H .
$$

Then $P_{V}$ is the orthogonal projection of $H$ on $V$. Let $G:=\left.P_{V}\right|_{\widetilde{V}}$ be the restriction of $P_{V}$ to $\widetilde{V}$. If $f \in \widetilde{V}$ and $G(f)=0$, then $f \in \widetilde{V} \cap V^{\perp}=\{0\}$. So $G$ is injective, and

$$
G(\widetilde{V})=P_{V}(\widetilde{V})=P_{V}\left(\widetilde{V}+V^{\perp}\right)=P_{V}(H)=V .
$$

Hence $G$ is an invertible bounded operator from $\widetilde{V}$ onto $V$. Define

$$
\tilde{v}_{n}=G^{-1}\left(u_{n}\right), \quad \forall n .
$$

Then $\left\{\tilde{v}_{n}\right\}$ is a Riesz basis of $\widetilde{V}$, and for every $n$,

$$
u_{n}=G\left(\tilde{v}_{n}\right)=\sum_{k}\left\langle\tilde{v}_{n}, v_{k}\right\rangle u_{k} .
$$

Hence $\left\langle\tilde{v}_{n}, v_{k}\right\rangle=\delta_{n, k}, \forall n, k$. Therefore (ii) $\Longleftrightarrow$ (iii).

Interchanging the roles of $V$ and $\widetilde{V}$, we also have (i) $\Longleftrightarrow$ (iii).

(i) $\Longrightarrow$ (iv): Suppose that (i) holds. Since $V+\widetilde{V}^{\perp}$ is closed and $V \cap \widetilde{V}^{\perp}=\{0\}$, by Theorem 2.1,

$$
\sup \left\{|\langle v, w\rangle|: v \in V, w \in \widetilde{V}^{\perp},\|v\|=\|w\|=1\right\}<1 .
$$

Hence by $(2.2), \cos (\theta(V, \widetilde{V}))>0$. Since (ii) also holds, interchanging the roles of $V$ and $\widetilde{V}$ in the above argument, $\cos (\theta(\widetilde{V}, V))>0$, too.

(iv) $\Longrightarrow$ (i): Suppose that (iv) holds. Since $\cos (\theta(V, \widetilde{V}))>0$,

$$
\sup \left\{|\langle v, w\rangle|: v \in V, w \in \widetilde{V}^{\perp},\|v\|=\|w\|=1\right\}<1 .
$$

By Theorem 2.1, $V+\widetilde{V}^{\perp}$ is closed and $V \cap \widetilde{V}^{\perp}=\{0\}$. Since $\cos (\theta(\widetilde{V}, V))>0$ also, by the above argument, $\widetilde{V}+V^{\perp}$ is closed and $\widetilde{V} \cap V^{\perp}=\{0\}$. Hence

$$
V+\widetilde{V}^{\perp}=\left(V+\widetilde{V}^{\perp}\right)^{\perp \perp}=\left(V^{\perp} \cap \widetilde{V}\right)^{\perp}=H .
$$

Let $U=\left(U_{1}, \ldots, U_{d}\right)$ be an ordered $d$-tuple of distinct unitary operators on a Hilbert space $H$ such that $U_{k} U_{j}=U_{j} U_{k}, k, j=1, \ldots, d$. We shall use the multiindex notation $U^{m}=U_{1}^{m_{1}} \cdots U_{d}^{m_{d}}$ for $m=\left(m_{1}, \ldots, m_{d}\right) \in \mathbf{Z}^{d}$, with the convention that $U_{j}^{0}$ is the identity operator on $H, j=1, \ldots, d$. We also assume that $U^{m}$ is the identity operator only if $m=0$.

Corollary 2.4. Suppose that one of the conditions in Theorem 2.3 holds for the closed linear subspaces $V$ and $\widetilde{V}$ of $H$.

(a) Given a Riesz basis $\left\{v_{n}\right\}$ for $V$, there exists a Riesz basis $\left\{\tilde{v}_{n}\right\}$ for $\tilde{V}$ such that $\left\{v_{n}\right\}$ is biorthogonal to $\left\{\tilde{v}_{n}\right\}$.

(b) If $\left\{U^{n} w_{j}: n \in \mathbf{Z}^{d}, j=1, \ldots, r\right\}$ is a Riesz basis for $V$ for some positive integer $r$, and

$$
U_{k}(\widetilde{V}) \subset \widetilde{V}, \quad k=1, \ldots, d,
$$

then there exist $\tilde{w}_{1}, \ldots, \tilde{w}_{r}$ in $\widetilde{V}$ such that $\left\{U^{n} \tilde{w}_{j}: n \in \mathbf{Z}^{d}, j=1, \ldots, r\right\}$ is a Riesz basis for $\widetilde{V}$, and it is biorthogonal to $\left\{U^{n} w_{j}: n \in \mathbf{Z}^{d}, j=1, \ldots, r\right\}$. 
Proof. We follow the proof of the implication (ii) $\Longrightarrow$ (iii) in Theorem 2.3, which gives (a) directly.

For (b), suppose now that $V$ has a Riesz basis of the form $\left\{U^{n} w_{j}: n \in \mathbf{Z}^{d}, j=\right.$ $1, \ldots, r\}$. By $(2.7), F$ commutes with each $U_{k}, k=1, \ldots, d$. Using this observation and (2.8), the dual Riesz basis in $V$ is given by $\left\{U^{n} z_{j}: n \in \mathbf{Z}^{d}, j=1, \ldots, r\right\}$, where $z_{j}=F^{-1}\left(w_{j}\right), j=1, \ldots, r$. Likewise, by (2.9), $G$ commutes with each $U_{k}, k=1, \ldots, d$. Using (2.10), $\left\{U^{n} \tilde{w}_{j}: n \in \mathbf{Z}^{d}, j=1, \ldots, r\right\}$ is then the desired Riesz basis for $\widetilde{V}$, where $\tilde{w}_{j}=G^{-1}\left(z_{j}\right), j=1, \ldots, r$.

Corollary 2.5 ([1, Theorem 3.2 (iii)]). Let $T$ be a unitary operator on $H$. Let $V$ and $W$ be closed linear subspaces of $H$, and let $\left\{T^{n} v_{j}: n \in \mathbf{Z}, j=1, \ldots, r\right\}$ and $\left\{T^{n} w_{j}: n \in \mathbf{Z}, j=1, \ldots, r\right\}$ be Riesz bases for $V$ and $W$ respectively, for some positive integer $r$. Then the oblique projection of $H$ on $V$ along $W^{\perp}$ is well defined (i.e., $V \oplus W^{\perp}=H$ ) if and only if $\cos (\theta(V, W))>0$.

Proof. This follows from Theorem 2.3 and the result [1, Theorem 3.2(i)] that for such $V$ and $W, \cos (\theta(V, W))=\cos (\theta(W, V))$.

Corollary 2.6. Let $V, \tilde{V}, W$ and $\tilde{W}$ be closed linear subspaces of $H$, and let $V \perp \tilde{W}$ and $W \perp \tilde{V}$. Let $\left\{v_{n}\right\},\left\{\tilde{v}_{n}\right\},\left\{w_{n}\right\}$, and $\left\{\tilde{w}_{n}\right\}$ be Riesz bases for $V, \tilde{V}, W$ and $\tilde{W}$ respectively, let $\left\{v_{n}\right\}$ be biorthogonal to $\left\{\tilde{v}_{n}\right\}$, and let $\left\{w_{n}\right\}$ be biorthogonal to $\left\{\tilde{w}_{n}\right\}$. Then $V \cap W=\{0\}, V+W$ is closed, and $\left\{v_{n}\right\} \cup\left\{w_{n}\right\}$ is a Riesz basis for $V \oplus W$.

Proof. By Theorem 2.3, $V \cap \widetilde{V}^{\perp}=\{0\}$, and $V+\widetilde{V}^{\perp}=H$. In particular, $V+\widetilde{V}^{\perp}$ is closed. Since $W \subset \widetilde{V}^{\perp}$, we have $V \cap W=\{0\}$, and by Corollary 2.2, $V+W$ is closed. Hence the desired result follows from Theorem 2.1.

\section{An EXTENSION THEOREM AND BIORTHOGONAL MULtiWAVELEtS}

Let $V_{0}, \widetilde{V}_{0}, V_{1}$ and $\widetilde{V}_{1}$ be closed linear subspaces of a Hilbert space $H$, let $V_{0} \subset$ $V_{1}, \widetilde{V}_{0} \subset \widetilde{V}_{1}$, and for $i=0,1$, let $V_{i}$ and $\widetilde{V}_{i}$ have biorthogonal Riesz bases. By Theorem 2.3,

$$
V_{0} \cap \widetilde{V}_{0}^{\perp}=\{0\}, \quad V_{0}+\widetilde{V}_{0}^{\perp}=H
$$

and

$$
V_{1} \cap \widetilde{V}_{1}^{\perp}=\{0\}, \quad V_{1}+\widetilde{V}_{1}^{\perp}=H
$$

Define

$$
W_{0}=V_{1} \cap \widetilde{V}_{0}^{\perp}
$$

and

$$
\widetilde{W}_{0}=\widetilde{V}_{1} \cap V_{0}^{\perp}
$$

We collect below some properties of $W_{0}$ and $\widetilde{W}_{0}$.

Proposition 3.1. $W_{0}$ and $\widetilde{W}_{0}$ have the following properties:

(i) $\quad W_{0}$ is the unique closed linear subspace of $H$ satisfying the conditions $V_{1}=$ $V_{0} \oplus W_{0}$ and $W_{0} \perp \widetilde{V}_{0}$.

(ii) $\quad \widetilde{W}_{0}^{\perp}=V_{0}+\widetilde{V}_{1}^{\perp}$.

(iii) $W_{0} \oplus \widetilde{W}_{0}^{\perp}=H$.

(iv) If $P, Q$ and $R$ are the projection on $V_{1}$ along $\widetilde{V}_{1}^{\perp}$, the projection on $V_{0}$ along $\widetilde{V}_{0}^{\perp}$, and the projection on $W_{0}$ along $\widetilde{W}_{0}^{\perp}$ respectively, then $P=Q+R$. 
Proof. We omit the proof of (i), which follows from some simple arguments using (3.1) and (3.3).

Since $V_{0} \subset V_{1}$, by (3.2) and Corollary 2.2, $V_{0}+\widetilde{V}_{1}^{\perp}$ is closed. Hence

$$
\widetilde{W}_{0}^{\perp}=\left(\widetilde{V}_{1} \cap V_{0}^{\perp}\right)^{\perp}=\overline{\widetilde{V}_{1}^{\perp}+V_{0}}=V_{0}+\widetilde{V}_{1}^{\perp} .
$$

Now $W_{0} \cap \widetilde{W}_{0}^{\perp}=\left(V_{1} \cap \widetilde{V}_{0}^{\perp}\right) \cap\left(V_{0}+\widetilde{V}_{1}^{\perp}\right)$. Let $x \in V_{1} \cap \widetilde{V}_{0}^{\perp}$ and $x=v+w$ for some $v$ in $V_{0}$ and $w$ in $\widetilde{V}_{1}^{\perp}$. Then $w$ is in $\widetilde{V}_{0}^{\perp}$, and $v=x-w \in \widetilde{V}_{0}^{\perp} \cap V_{0}=\{0\}$. Hence $x=w \in V_{1} \cap \widetilde{V}_{1}^{\perp}=\{0\}$. Thus $W_{0} \cap \widetilde{W}_{0}^{\perp}=\{0\}$, and

$$
W_{0}+\widetilde{W}_{0}^{\perp}=W_{0}+\left(V_{0}+\widetilde{V}_{1}^{\perp}\right)=V_{1}+\widetilde{V}_{1}^{\perp}=H .
$$

Finally, (iv) is an easy consequence of the above properties.

We recall some notation and terminology from [6]. Let $U:=\left(U_{1}, \ldots, U_{d}\right)$ be an ordered $d$-tuple of commuting distinct unitary operators on a Hilbert space $H$. For a subset $S$ of $H$, let $\langle S\rangle$ denote the closed linear span of $S$, and

$$
U^{\mathbf{Z}^{d}}(S):=\left\{U^{n} s: n \in \mathbf{Z}^{d}, s \in S\right\} .
$$

If $V=\left\{v_{1}, \ldots, v_{r}\right\}$ and $W=\left\{w_{1}, \ldots, w_{p}\right\}$ are finite subsets of $H$ such that

$$
\left\{\left\langle v_{k}, U^{n} w_{j}\right\rangle\right\}_{n \in \mathbf{Z}^{d}} \in \ell^{2}\left(\mathbf{Z}^{d}\right), \quad k=1, \ldots, r, j=1, \ldots, p,
$$

then the function $\Phi_{V, W}$ defined almost everywhere on $\mathbf{R}^{d}$ by

$$
\Phi_{V, W}(\theta):=\left(\sum_{n \in \mathbf{Z}^{d}}\left\langle v_{k}, U^{n} w_{j}\right\rangle e^{i n \cdot \theta}\right)_{1 \leq k \leq r, 1 \leq j \leq p}
$$

is an $r \times p$ matrix function with entries in $L^{2}\left([0,2 \pi)^{d}\right)$. It is easy to verify the following properties:

$$
\begin{gathered}
\left(\Phi_{V, W}(\theta)\right)^{*}=\Phi_{W, V}(\theta) ; \\
V \perp U^{\mathbf{Z}^{d}}(W) \Longleftrightarrow \Phi_{V, W}(\theta)=0 \text { a.e.; }
\end{gathered}
$$

and if $r=p$, then

$$
\left\langle U^{n} v_{k}, U^{m} w_{j}\right\rangle=\delta_{n, m} \delta_{k, j}, k, j=1, \ldots, r, n, m \in \mathbf{Z}^{d} \Longleftrightarrow \Phi_{V, W}(\theta)=I_{r} \text { a.e., }
$$

where $I_{r}$ is the $r \times r$ identity matrix.

A deeper result is the following [6, Theorem 2.1]:

Theorem 3.2. If a finite subset $V=\left\{v_{1}, \ldots, v_{r}\right\}$ of $H$ satisfies

$$
\sum_{n \in \mathbf{Z}^{d}}\left|\left\langle v_{k}, U^{n} v_{j}\right\rangle\right|^{2}<\infty, \quad k, j=1, \ldots, r,
$$

then $U^{\mathbf{Z}^{d}}(V)$ is a Riesz basis for $\left\langle U^{\mathbf{Z}^{d}}(V)\right\rangle$ if and only if there exist positive constants $C_{1}$ and $C_{2}$ such that

$$
C_{1} \leq \Phi_{V, V}(\theta) \leq C_{2}
$$

for almost every $\theta \in \mathbf{R}^{d}$. 
Assume now that $Y=\left\{y_{1}, \ldots, y_{s}\right\}$ and $\widetilde{Y}=\left\{\tilde{y}_{1}, \ldots, \tilde{y}_{s}\right\}$ are finite subsets of $H$ such that $U^{\mathbf{Z}^{d}}(Y)$ and $U^{\mathbf{Z}^{d}}(\tilde{Y})$ are Riesz bases for $\left\langle U^{\mathbf{Z}^{d}}(Y)\right\rangle$ and $\left\langle U^{\mathbf{Z}^{d}}(\widetilde{Y})\right\rangle$ respectively, and $U^{\mathbf{Z}^{d}}(Y)$ and $U^{\mathbf{Z}^{d}}(\widetilde{Y})$ are biorthogonal, i.e.,

$$
\left\langle U^{n} y_{k}, U^{m} \tilde{y}_{j}\right\rangle=\delta_{n, m} \delta_{k, j}, \quad k, j=1, \ldots, s, \quad n, m \in \mathbf{Z}^{d} .
$$

In particular, since $U^{\mathbf{Z}^{d}}(Y)$ and $U^{\mathbf{Z}^{d}}(\tilde{Y})$ are Bessel sequences in $H$ (see [12, pp. 154-155]), the entries of the matrix functions $\Phi_{V, Y}$ and $\Phi_{V, \widetilde{Y}}$ are $L^{2}$-functions for any finite subset $V$ of $H$. By (3.9) and (3.12),

$$
\Phi_{Y, \tilde{Y}}=\Phi_{\tilde{Y}, Y}=I_{s} \text { a.e., }
$$

where $I_{s}$ is the $s \times s$ identity matrix, and we have the biorthogonal expansions

$$
f=\sum_{j=1}^{s} \sum_{n \in \mathbf{Z}^{d}}\left\langle f, U^{n} \tilde{y}_{j}\right\rangle U^{n} y_{j} \quad f \in\left\langle U^{\mathbf{Z}^{d}}(Y)\right\rangle,
$$

and

$$
g=\sum_{j=1}^{s} \sum_{n \in \mathbf{Z}^{d}}\left\langle g, U^{n} y_{j}\right\rangle U^{n} \tilde{y}_{j}, \quad g \in\left\langle U^{\mathbf{Z}^{d}}(\widetilde{Y})\right\rangle .
$$

We also have the following results.

Proposition 3.3. Let $V=\left\{v_{1}, \ldots, v_{r}\right\}$ and $W=\left\{w_{1}, \ldots, w_{p}\right\}$ be finite subsets of $H$ satisfying condition (3.5).

$$
\begin{aligned}
& \text { If } V \subset\left\langle U^{\left.\mathbf{Z}^{d}(Y)\right\rangle \text {, then }}\right. \\
& \qquad \Phi_{V, W}=\Phi_{V, \widetilde{Y}} \Phi_{W, Y}^{*}, \quad \Phi_{W, V}=\Phi_{W, Y} \Phi_{V, \widetilde{Y}}^{*} \\
& \text { and }
\end{aligned}
$$

(ii) If $V \subset\left\langle U^{\mathbf{Z}^{d}}(\widetilde{Y})\right\rangle$, then

$$
\Phi_{V, W}=\Phi_{V, Y} \Phi_{W, \tilde{Y}}^{*}, \quad \Phi_{W, V}=\Phi_{W, \widetilde{Y}} \Phi_{V, Y}^{*}
$$

(iii) If $V \subset\left\langle U^{\mathbf{Z}^{d}}(Y)\right\rangle$ and condition (3.10) holds, then

$$
\Phi_{V, V}=\Phi_{V, Y} \Phi_{V, \widetilde{Y}}^{*}=\Phi_{V, \widetilde{Y}} \Phi_{V, Y}^{*}=\Phi_{V, \widetilde{Y}} \Phi_{Y, Y} \Phi_{V, \tilde{Y}}^{*}
$$

Proof. By (3.14), for $\nu \in \mathbf{Z}^{d}, k=1, \ldots, r, \ell=1, \ldots, p$,

$$
\left\langle v_{k}, U^{\nu} w_{\ell}\right\rangle=\sum_{j=1}^{s} \sum_{n \in \mathbf{Z}^{d}}\left\langle v_{k}, U^{n} \tilde{y}_{j}\right\rangle\left\langle y_{j}, U^{\nu-n} w_{\ell}\right\rangle .
$$

Hence $\Phi_{V, W}=\Phi_{V, \widetilde{Y}} \Phi_{Y, W}=\Phi_{V, \widetilde{Y}} \Phi_{W, Y}^{*}$. The second equality in (3.16) follows by taking adjoints. For (3.17), take $W=Y$. (ii) is proved similarly using (3.15), and (iii) follows directly from (i).

We should emphasis that our present setting is more general than that of [6]. There we assumed that $\left\langle U^{\mathbf{Z}^{d}}(Y)\right\rangle=\left\langle U^{\mathbf{Z}^{d}}(\widetilde{Y})\right\rangle$, but here we don't. Hence some of the results in [6] may not be valid in this new setting.

Using Theorem 3.2 and Proposition 3.3 above, the proof of [9, Proposition 3.3] can be carried over verbatim for our present setting to give 
Proposition 3.4. Let $V=\left\{v_{1}, \ldots, v_{r}\right\} \subset\left\langle U^{\mathbf{Z}^{d}}(Y)\right\rangle$, where $r \leq s$. The following conditions are equivalent:

(i) $\quad U^{\mathbf{Z}^{d}}(V)$ is a Riesz basis for $\left\langle U^{\mathbf{Z}^{d}}(V)\right\rangle$.

(ii) There exist positive constants $A$ and $B$ such that

$$
A \leq \Phi_{V, Y} \Phi_{V, Y}^{*} \leq B \text { a.e. }
$$

(iii) There exist positive constants $\widetilde{A}$ and $\widetilde{B}$ such that

$$
\widetilde{A} \leq \Phi_{V, \widetilde{Y}} \Phi_{V, \widetilde{Y}}^{*} \leq \widetilde{B} \text { a.e. }
$$

We record one more useful result from [6, Theorem 2.4] (see also [9, Theorem 3.1]) that can still be applied here.

Theorem 3.5. Let $V=\left\{v_{1}, \ldots, v_{r}\right\}$ and $Y=\left\{y_{1}, \ldots, y_{s}\right\}$ be finite subsets of $H$ and suppose that $U^{\mathbf{Z}^{d}}(V)$ and $U^{\mathbf{Z}^{d}}(Y)$ are Riesz bases for $\left\langle U^{\mathbf{Z}^{d}}(V)\right\rangle$ and $\left\langle U^{\mathbf{Z}^{d}}(Y)\right\rangle$ respectively. If $\left\langle U^{\mathbf{Z}^{d}}(V)\right\rangle \subset\left\langle U^{\mathbf{Z}^{d}}(Y)\right\rangle$ and $r=s$, then $\left\langle U^{\mathbf{Z}^{d}}(V)\right\rangle=\left\langle U^{\mathbf{Z}^{d}}(Y)\right\rangle$.

We now state and prove the main result of this section, which is an extension of [6, Theorem 2.5] to the biorthogonal setting.

Theorem 3.6. Let $X=\left\{x_{1}, \ldots, x_{r}\right\}, \tilde{X}=\left\{\tilde{x}_{1}, \ldots, \tilde{x}_{r}\right\}, Y=\left\{y_{1}, \ldots, y_{s}\right\}$ and $\tilde{Y}=$ $\left\{\tilde{y}_{1}, \ldots, \tilde{y}_{s}\right\}$ be finite subsets of $H$. Let $U^{\mathbf{Z}^{d}}(X), U^{\mathbf{Z}^{d}}(\tilde{X}), U^{\mathbf{Z}^{d}}(Y)$ and $U^{\mathbf{Z}^{d}}(\tilde{Y})$ be Riesz bases for their closed linear spans $V_{0}, \widetilde{V}_{0}, V_{1}$ and $\widetilde{V}_{1}$ respectively, $U^{\mathbf{Z}^{d}}(X)$ biorthogonal to $U^{\mathbf{Z}^{d}}(\widetilde{X}), U^{\mathbf{Z}^{d}}(Y)$ biorthogonal to $U^{\mathbf{Z}^{d}}(\widetilde{Y})$, and

$$
V_{0} \subset V_{1}, \quad \widetilde{V}_{0} \subset \widetilde{V}_{1} .
$$

Let $W_{0}=V_{1} \cap \widetilde{V}_{0}^{\perp}$ and $\widetilde{W}_{0}=\widetilde{V}_{1} \cap V_{0}^{\perp}$. If $r<s$, then

(i) there exists a subset $\Gamma:=\left\{z_{1}, \ldots, z_{s-r}\right\}$ of $W_{0}$ such that $U^{\mathbf{Z}^{d}}(\Gamma)$ is a Riesz basis for $W_{0}$ and $U^{\mathbf{Z}^{d}}(X \cup \Gamma)$ is a Riesz basis for $V_{1}$, and

(ii) there exists a subset $\widetilde{\Gamma}:=\left\{\tilde{z}_{1}, \ldots, \tilde{z}_{s-r}\right\}$ of $\widetilde{W}_{0}$ such that $U^{\mathbf{Z}^{d}}(\widetilde{\Gamma})$ is a Riesz basis for $\widetilde{W}_{0}$ and $U^{\mathbf{Z}^{d}}(\widetilde{X} \cup \widetilde{\Gamma})$ is a Riesz basis for $\widetilde{V}_{1}$, and $U^{\mathbf{Z}^{d}}(\widetilde{\Gamma})$ is biorthogonal to $U^{\mathbf{Z}^{d}}(\Gamma)$.

Proof. Since the $r \times s$ matrix $\Phi_{\widetilde{X}, Y}(\theta)$ has at most rank $r$, choose an $(s-r) \times s$ matrix $Y(\theta)$ such that

$$
Y(\theta) \Phi_{\widetilde{X}, Y}(\theta)^{*}=0
$$

and

$$
Y(\theta) Y(\theta)^{*}=I_{s-r} .
$$

(The measurability of one such function $Y$ is assured by some standard arguments in measure theory; see, e.g., [8, Lemma 2.4].) Let the $(k, j)$-entry of $Y(\theta)$ be $Y_{k, j}(\theta), k=1, \ldots, s-r, j=1, \ldots, s$. By $(3.21)$,

$$
\sum_{j=1}^{s}\left|Y_{k, j}(\theta)\right|^{2}=1, \quad k=1, \ldots, s-r,
$$

and so all $Y_{k, j}$ are bounded functions in $L^{2}\left([0,2 \pi)^{d}\right)$. For $k=1, \ldots, s-r, j=1, \ldots, s$, let

$$
Y_{k, j}(\theta)=\sum_{n \in \mathbf{Z}^{d}} a_{k, j}(n) e^{i n \cdot \theta}
$$


where $\left\{a_{k, j}(n)\right\}_{n \in \mathbf{Z}^{d}} \in \ell^{2}\left(\mathbf{Z}^{d}\right)$, and let

$$
z_{k}=\sum_{j=1}^{s} \sum_{n \in \mathbf{Z}^{d}} a_{k, j}(n) U^{n} y_{j} .
$$

Then $z_{k}$ is in $V_{1}$, and by (3.22), (3.23) and (3.6),

$$
Y(\theta)=\Phi_{\Gamma, \tilde{Y}}(\theta) \quad \text { a.e. }
$$

where $\Gamma:=\left\{z_{1}, \ldots, z_{s-r}\right\}$. By (3.16) and (3.20),

$$
\Phi_{\Gamma, \widetilde{X}}=\Phi_{\Gamma, \widetilde{Y}} \Phi_{\widetilde{X}, Y}^{*}=0 \quad \text { a.e. }
$$

Hence by (3.8), $\Gamma \perp U^{\mathbf{Z}^{d}}(\tilde{X})$. By (3.21) and (3.24),

$$
\Phi_{\Gamma, \tilde{Y}} \Phi_{\Gamma, \tilde{Y}}^{*}=I_{s-r} \text { a.e. }
$$

Hence by Proposition 3.4, $U^{\mathbf{Z}^{d}}(\Gamma)$ is a Riesz basis for $\left\langle U^{\mathbf{Z}^{d}}(\Gamma)\right\rangle$.

By Proposition 3.1, $V_{0} \oplus W_{0}=V_{1}$, which is closed in $H$. Since

$$
\left\langle U^{\mathbf{Z}^{d}}(\Gamma)\right\rangle \subset V_{1} \cap \widetilde{V}_{0}^{\perp}=W_{0},
$$

using Corollary 2.2, $V_{0}+\left\langle U^{\mathbf{Z}^{d}}(\Gamma)\right\rangle$ is also closed in $H$ and $V_{0} \cap\left\langle U^{\mathbf{Z}^{d}}(\Gamma)\right\rangle=\{0\}$. Hence by Theorem 2.1, $U^{\mathbf{Z}^{d}}(X \cup \Gamma)$ is a Riesz basis for $V_{0}+\left\langle U^{\mathbf{Z}^{d}}(\Gamma)\right\rangle$. Since

$$
\left\langle U^{\mathbf{Z}^{d}}(X \cup \Gamma)\right\rangle=V_{0}+\left\langle U^{\mathbf{Z}^{d}}(\Gamma)\right\rangle \subset V_{1}=\left\langle U^{\mathbf{Z}^{d}}(Y)\right\rangle
$$

and $\#(X \cup \Gamma)=\#(Y)=s$, by Theorem 3.5 we have

$$
V_{0} \oplus\left\langle U^{\mathbf{Z}^{d}}(\Gamma)\right\rangle=V_{1}=V_{0} \oplus W_{0} .
$$

Since $\left\langle U^{\mathbf{Z}^{d}}(\Gamma)\right\rangle \subset W_{0}$, we then actually have $\left\langle U^{\mathbf{Z}^{d}}(\Gamma)\right\rangle=W_{0}$, and as proven above, $U^{\mathbf{Z}^{d}}(X \cup \Gamma)$ is a Riesz basis for $V_{1}$. This completes the proof of (i).

For each $k=1, \ldots, d$, since $U_{k}$ is unitary, $U_{k}\left(V_{1}\right)=V_{1}$, and $U_{k}\left(\widetilde{V}_{0}\right)=\widetilde{V}_{0}$, we have $U_{k}\left(\widetilde{W}_{0}\right)=\widetilde{W}_{0}$. By Proposition 3.1, $W_{0} \oplus \widetilde{W}_{0}^{\perp}=H$. Hence the results of (ii) follow from (i), Corollary 2.4 and Theorem 2.1 .

Remark 3.7. Letting $V_{0}=\widetilde{V}_{0}$ and $V_{1}=\widetilde{V}_{1}$ in Theorem 3.6, we recover the results of $\left[6\right.$, Theorem 2.5]. In this case, $W_{0}=\widetilde{W}_{0}$, and $V_{1}$ is the orthogonal direct sum of $V_{0}$ and $W_{0}$.

\section{Biorthogonal Wavelets}

We follow the notation in Section 3. Let $U:=\left(U_{1}, \ldots, U_{d}\right)$ be an ordered $d$-tuple of commuting distinct unitary operators on a Hilbert space $H$. Let $X=\left\{x_{1}, \ldots, x_{r}\right\}$ and $\tilde{X}=\left\{\tilde{x}_{1}, \ldots, \tilde{x}_{r}\right\}$ be finite subsets of $H$ such that $U^{\mathbf{Z}^{d}}(X)$ and $U^{\mathbf{Z}^{d}}(\tilde{X})$ are biorthogonal Riesz bases for their closed linear spans $V_{0}$ and $\widetilde{V}_{0}$ respectively. Now suppose that there is a unitary operator $D$ on $H$ such that

$$
V_{0} \subset V_{1}:=D\left(V_{0}\right), \quad \widetilde{V}_{0} \subset \widetilde{V}_{1}:=D\left(\widetilde{V}_{0}\right)
$$

and

$$
U^{n} D=D U^{M n}, \quad n \in \mathbf{Z}^{d}
$$

where $M$ is a $d \times d$ matrix with integer entries and $|\operatorname{det}(M)|>1$. Under this setting, Theorem 3.6 yields analogous results to parts (1) and (2) of [6, Theorem 
3.1], leading to the existence of biorthogonal multiwavelets in the Hilbert space setting. A special case of this is when $H=L^{2}\left(\mathbf{R}^{d}\right)$,

$$
\left(U_{k} f\right)(x)=f\left(x-e_{k}\right),
$$

where $e_{k}=\left(\delta_{k, j}\right)_{j=1, \ldots, d}, k=1, \ldots, d$, and

$$
(D f)(x)=|\operatorname{det}(M)|^{\frac{1}{2}} f(M x),
$$

for $x$ in $\mathbf{R}^{d}$ and $f$ in $L^{2}\left(\mathbf{R}^{d}\right)$. We shall not go into this any further. Instead, let us consider the special case when $d=1, r=1$ and $s=2$.

Let $T$ and $D$ be unitary operators on a Hilbert space $H$ such that

$$
T D=D T^{2} .
$$

Let $T^{\mathbf{Z}}(\{\phi\})$ and $T^{\mathbf{Z}}(\{\tilde{\phi}\})$ be biorthogonal Riesz bases for their closed linear spans $V_{0}$ and $\widetilde{V}_{0}$ respectively such that $(4.1)$ holds. For $j=0,1$, let $\phi_{j}:=D T^{j} \phi$ and $\tilde{\phi}_{j}:=D T^{j} \tilde{\phi}$. Then

$$
T^{\mathbf{Z}}\left(\left\{\phi_{0}, \phi_{1}\right\}\right)=\left\{D T^{n} \phi: n \in \mathbf{Z}\right\}
$$

and

$$
T^{\mathbf{Z}}\left(\left\{\tilde{\phi}_{0}, \tilde{\phi}_{1}\right\}\right)=\left\{D T^{n} \tilde{\phi}: n \in \mathbf{Z}\right\}
$$

are biorthogonal Riesz bases for $V_{1}$ and $\widetilde{V}_{1}$ respectively. Since $\phi$ is in $V_{1}$ and $\tilde{\phi}$ is in $\widetilde{V}_{1}$,

$$
\phi=\sum_{n \in \mathbf{Z}} \tilde{c}_{n} D T^{n} \phi
$$

and

$$
\tilde{\phi}=\sum_{n \in \mathbf{Z}} d_{n} D T^{n} \tilde{\phi}
$$

for some sequences $\left\{\tilde{c}_{n}\right\}$ and $\left\{d_{n}\right\}$ in $\ell^{2}(\mathbf{Z})$.

Theorem 4.1. Let

$$
\eta:=\sum_{n \in \mathbf{Z}}(-1)^{-n+1} \overline{d_{-n+1}} D T^{n} \phi
$$

and

$$
\tilde{\eta}:=\sum_{n \in \mathbf{Z}}(-1)^{-n+1} \overline{\tilde{c}_{-n+1}} D T^{n} \tilde{\phi} .
$$

Then $T^{\mathbf{Z}}(\{\eta\})$ and $T^{\mathbf{Z}}(\{\tilde{\eta}\})$ are biorthogonal Riesz bases for $W_{0}:=V_{1} \cap \widetilde{V}_{0}^{\perp}$ and $\widetilde{W}_{0}:=\widetilde{V}_{1} \cap V_{0}^{\perp}$ respectively.

We omit the details here. The proof is a minor modification of the arguments in Section 4 of [9], by first establishing analogous results for our present biorthogonal setting.

Remark 4.2. If $H=L^{2}(\mathbf{R}), T f(x)=f(x-1)$, and $D f(x)=\sqrt{2} f(2 x)$, for $x$ in $\mathbf{R}$ and $f$ in $L^{2}(\mathbf{R})$, then Theorem 4.1 recovers the explicit formulae of the biorthogonal wavelets, constructed in [2], that are associated with biorthogonal scaling functions. 


\section{ACKNOWLEDGEMENT}

This research was supported by the Wavelets Strategic Research Programme, National University of Singapore, under a grant from the National Science and Technology Board and the Ministry of Education, Republic of Singapore. We would like to thank Professor S. L. Lee for his many helpful suggestions on this paper. After the completion of the first draft of this manuscript, Professor T. N. T. Goodman communicated orally to us that he and his co-authors had also obtained parts of Theorem 2.1 of this paper.

\section{REFERENCES}

[1] A. Aldroubi, Oblique projections in atomic spaces, Proc. Amer. Math. Soc. 124 (1996), 20512060. MR 96i:42020

[2] A. Cohen, I. Daubechies and J. C. Feauveau, Biorthogonal bases of compactly supported wavelets, Comm. Pure Appl. Math. XLV (1992), 485-560. MR 93e:42044

[3] X. Dai and D. R. Larson, Wandering vectors for unitary systems and orthogonal wavelets, Mem. Amer. Math. Soc. 134 (1998), no. 640. MR 98m:47067

[4] I. C. Gohberg and M. G. Krein, Introduction to The Theory of Linear Nonselfadjoint Operators, Translations of Mathematical Monographs, Vol. 18, American Mathematical Society, Providence, 1969. MR 39:7447

[5] T. N. T. Goodman, S. L. Lee and W. S. Tang, Wavelets in wandering subspaces, Trans. Amer. Math. Soc. 338 (1993), 639-654. MR 93j:42017

[6] T. N. T. Goodman, S. L. Lee and W. S. Tang, Wavelet bases for a set of commuting unitary operators, Adv. Comput. Math. 1 (1993), 109-126. MR 94h:42057

[7] P. R. Halmos, Introduction to Hilbert Spaces and Spectral Multiplicity, Chelsea, New York, 1951. MR 13:563a

[8] R. Q. Jia and Z. W. Shen, Multiresolution and wavelets, Proc. Edinburgh Math. Soc. 37 (1994), 271-300. MR 95h:42035

[9] S. L. Lee, H. H. Tan and W. S. Tang, Wavelet bases for a unitary operator, Proc. Edinburgh Math. Soc. 38 (1995), 233-260. MR 96g:42019

[10] J. B. Robertson, On wandering subspaces for unitary operators, Proc. Amer. Math. Soc. 16 (1965), 233-236. MR 30:5165

[11] M. Unser and A. Aldroubi, A general sampling theory for non-ideal acquisition devices, IEEE Trans. on Signal Processing 42 (1994), 2915-2925.

[12] R. M. Young, An Introduction to Nonharmonic Fourier Series, Academic Press, New York, 1980. MR 81m:42027

Department of Mathematics, National University of Singapore, 10 Kent Ridge CresCEnt, 119260, Republic of Singapore

E-mail address: mattws@math.nus.edu.sg 